\title{
Finding of left atrial appendage thrombus despite a normal preoperative transthoracic echocardiography. Importance of intraoperative TEE. Case report
}

\section{Sara López Palanca ${ }^{1}$, Eva Mateo Rodríguez ${ }^{2}$, Paula Carmona García ${ }^{3}$, Ana María Rodilla Fiz ${ }^{4}$}

${ }^{1}$ Anesthesia and Intensive Care. General Hospital of Albacete. Albacete. Spain. ${ }^{2}$ Cardiovascular-Anesthesia and Intensive Care. General Hospital of Valencia. Valencia. Spain. ${ }^{3}$ Cardiovascular-Anesthesia and Intensive Care. University Hospital La Fe. Valencia. Spain. ${ }^{4}$ Anesthesia and Intensive Care. General Hospital of Albacete. Albacete. Spain

\section{Background:}

- Transthoracic echocardiography (TTE) cannot reliably exclude left atrial (LA) thrombi prior cardiac surgery

- LA trombi have been shown to be associated with an increased stroke rate

- TEE has a sensitive and specificity of $100 \%$ in detection of LA and left atrial appendage (LAA) thrombi in contrast to TTE which has limitations when assessing the presence of masses or thrombi in the left atrium.

\section{Case Report:}

- Woman 60 years old scheduled for aortic and mitral replacement. Her history includes atrial fibrillation and ischemic stroke

- Chronic anticoagulation was suspended five days before surgery and low molecular weight heparin began

- The intraoperative TEE revealed a new $20 \times 30 \mathrm{~mm}$ diameter pedunculated mass in LAA protruding to LA

- Preoperative TTE had been performed 2 months before, showing any mass inside
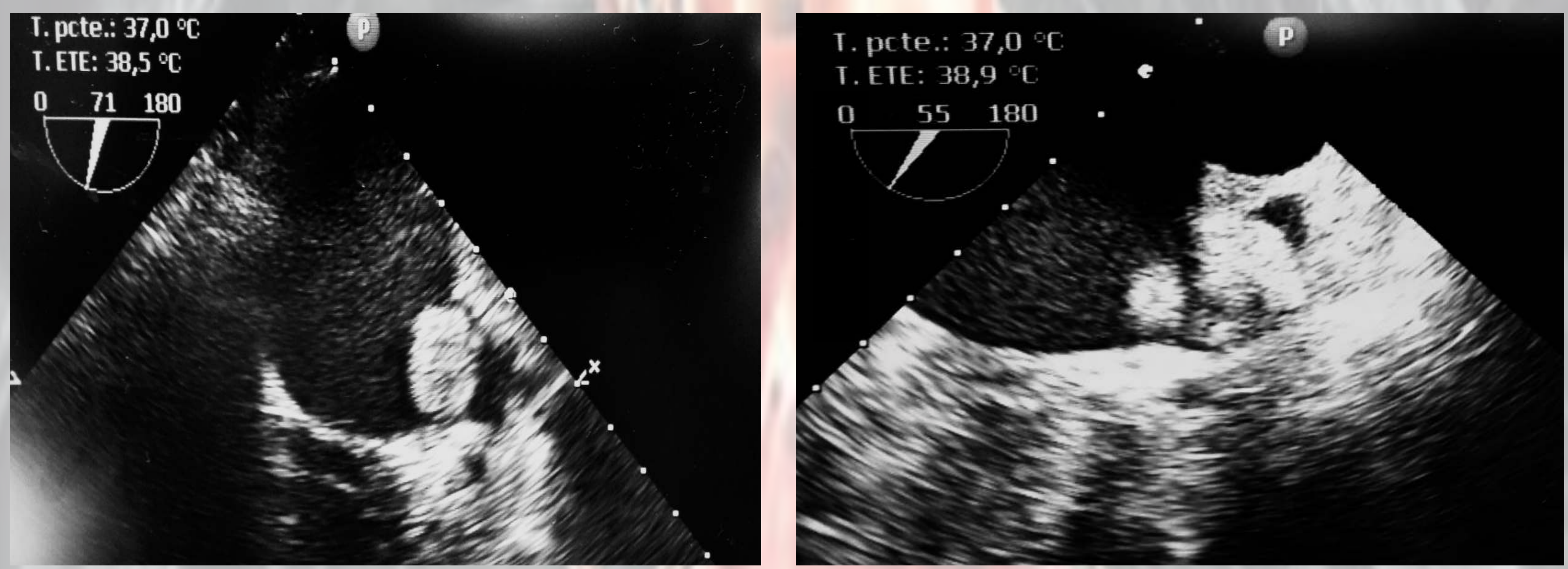

\section{Discussion:}

- According to the latest guidelines, TEE should be used in all open heart procedures in order to: (1) confirm and refine the preoperative diagnosis, (2) detect new or unsuspected pathology, (3) adjust the anesthetic and surgical plan accordingly, and (4) assess the results of surgical intervention.

- Our case confirms the importance of detection of new findings prior the operation which changes the surgical procedure and planning.

\begin{tabular}{|c|c|c|}
\hline & TTE & TEE \\
\hline $\begin{array}{c}\text { Thrombus LA/LAA } \\
\text { detection }\end{array}$ & $+/-$ & ++++ \\
\hline
\end{tabular}

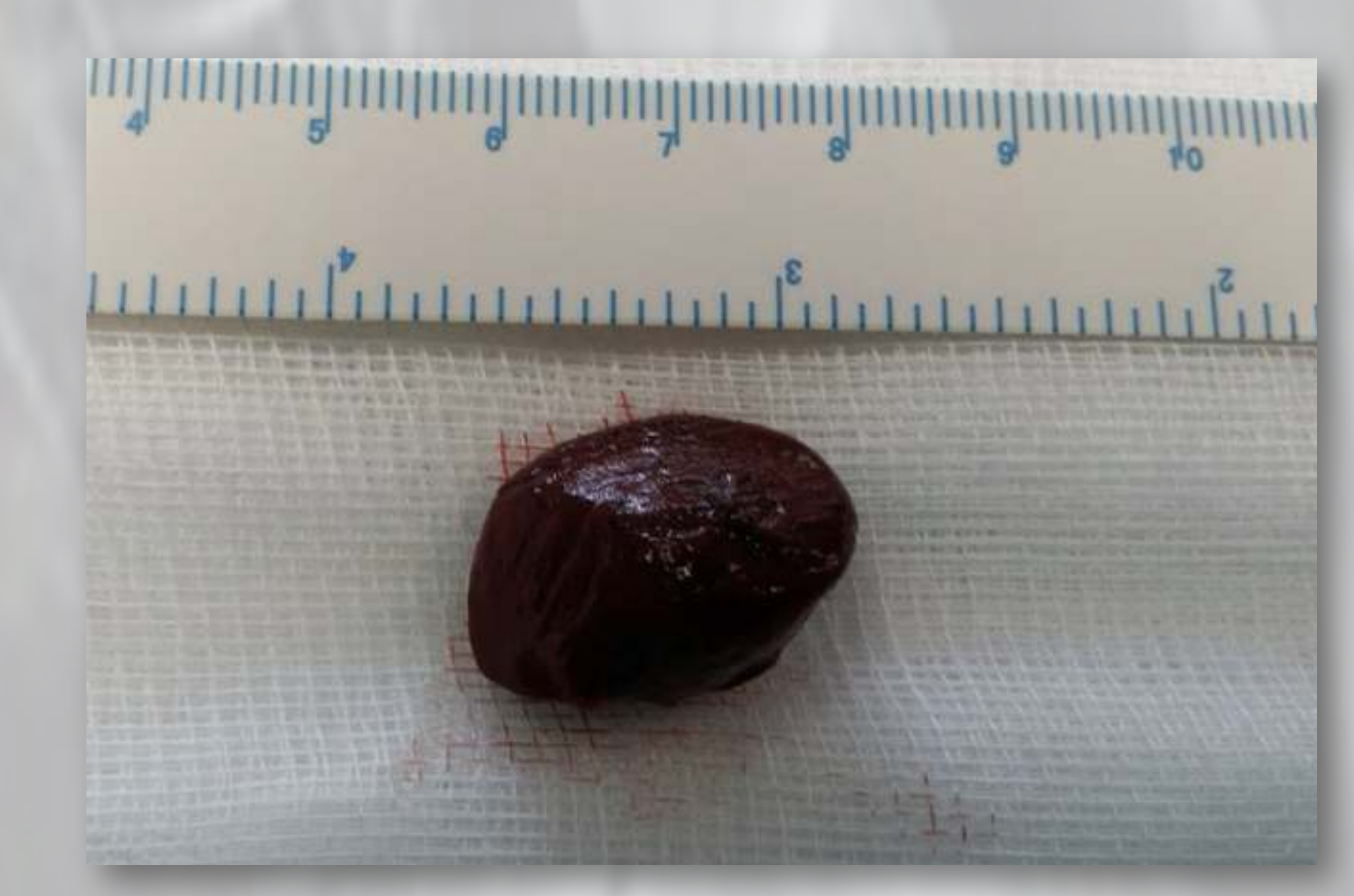

Thrombogenic risk increases with decreasing LAA velocities:

$><20 \mathrm{~cm} / \mathrm{s}(29 \%) 20-40 \mathrm{~cm} / \mathrm{s}(10 \%)$ and $>40 \mathrm{~cm} / \mathrm{s}(1 \%)$. The flow velocities in LAA as may incur indication surgery ligation

\section{Conclusions:}

- TEE should be used in all open heart procedures

- Intraoperative TEE gives the possibility to detect new pathologies that changes the surgical procedure.

1. Practice Guidelines for Perioperative Transesophageal Echocardiography. Anesthesiology 2010; 112:1084-96

2. ASE GUIDELINES. Guidelines for the Use of echocardiography in the Evaluation of a Cardiac Source of Embolism. J Am Soc Echocardiogr 2016;29:1-42.

3. Left atrial appendage occlusion. Alli O. et al. Heart 2015;101:834-841. 